\title{
El Portafolios como Herramienta Clave de la Evaluación Docente: Su Aplicación a las Asignaturas de Derecho del Trabajo en el Grado de Relaciones Laborales y Recursos Humanos
}

\author{
María José Gómez-Millán Herencia \\ María Dolores Mazuelos Flores \\ Rafael Gómez Gordillo*
}

Resumen:

El portafolios se ha convertido en uno de los instrumentos claves de los nuevos sistemas de enseñanza en el ámbito universitario, debido a las posibilidades que aporta en cuanto a la correcta evaluación de aquellas asignaturas donde el alumno es protagonista de su aprendizaje y su evaluación depende de las tareas realizadas durante el curso. Partiendo de esta idea, la presente comunicación analiza cómo se ha implantado en las asignaturas de Derecho del Trabajo pertenecientes al Grado de Relaciones Laborales $y$ Recursos Humanos de la Universidad Pablo de Olavide, a los efectos de explorar las oportunidades que ofrece como forma de evaluar las competencias de los alumnos.

Palabras Clave:

portafolios, sistema de evaluación, control del aprendizaje, métodos de enseñanza, organización de las clases, enseñanza superior, competencia, recursos de la enseñanza

ABstract:

Portfolios have become one of the instruments keys in new systems of higher education, due to the possibilities that contribute as far as the correct evaluation of those subjects where the student is protagonist of his learning and its evaluation depends on the tasks

* Departamento de Derecho del Trabajo y de la Seguridad Social. Universidad Pablo de Olavide • mjgommil@upo.es; mdmazflo@upo.es; rgomgor@upo.es 
carried out during the course. Starting off of this idea, this communication analyzes how it has been implanted at Labour Law's subjects pertaining to the Degree of Labour Relations and Human Resources at Pablo Olavide University, in order to explore the opportunities that offer like a tool wich it evaluate the competences of the students.

KEYWORDS:

Portfolios, student evaluation, learned controlled, teaching methods, class organization, bigher education, competence, resource teacher

\section{LAS DISTINTAS FORMAS DE EVALUAR AL ALUMNO: NUEVOS Y VIEJOS SISTEMAS}

La evaluación se puede definir, de manera general, como un proceso de recogida, análisis e interpretación de los conocimiento adquiridos por el alumno durante el transcurso de su aprendizaje. Un proceso que, frente a los conocimientos mínimos requeridos para superar la asignatura, permite calificar al alumno en función de parámetros objetivos que pueden tener como indicadores, bien conocimientos concretos relacionados con la asignatura, o bien habilidades profesionales relacionadas con la Titulación. En este sentido, conviene destacar que la evaluación es un proceso que consiste en "medir" o calificar conocimiento, haciendo uso de criterios o normas establecidas antes de iniciar el proceso formativo, lo que aporta cierta seguridad al alumno, en relación con los conocimientos que deben acreditar quienes superen la asignatura. Dicho lo anterior, resulta evidente que todo método de evaluación debe huir de elementos que aporten subjetividad, pues este proceso debe centrarse en medir los objetivos superados, siendo necesario que se recojan las pruebas de los logros que los alumnos han alcanzado durante todo el curso académico.

El proceso de evaluación, en efecto, puede referirse a los siguientes conceptos, que se relacionan con competencias específicas, cada una de las cuales son propias de cada Titulación: a) Los contenidos conceptuales, relacionados con las materias o contenidos básicos o mínimos de las asignaturas, de carácter más bien teórico, y relacionados con las partes fundamentales de la asignatura; b) los contenidos resolutivos o de procedimiento en la realización de las actividades, conectados con la puesta en práctica de conocimientos teóricos, empleando para ello las técnicas propia de cada una de las asignaturas o materias; c) los contenidos actitudinales o de comportamiento, relacionados con las habilidades personales y sociales de cada uno de los estudiantes que se consideran como necesarias o adecuadas en cada una de las Titulaciones.

Para evaluar estos contenidos, se pueden emplear, como se sabe, diferentes instrumentos, entre los que merece una consideración especial, por su orientación a cada uno de los conocimientos anteriores, los siguientes: En primer lugar, el examen 
se integra por preguntas de carácter teórico, práctico o teórico-práctico, en un proceso de evaluación donde el alumno se enfrenta a una prueba individual, quedando recogidos los resultados de forma oral o por escrito, para su posterior evaluación por el profesor. En ocasiones, el examen puede consistir en preguntas de tipo test, ya sean de tipo teórico o práctico, pudiendo tratarse de preguntas de verdadero/ falso, elección múltiple, abierta, que se evalúan por el profesor o por el alumno, en este último caso, a partir de la plantilla que el profesor entrega. En segundo lugar, el trabajo de investigación implica el desarrollo de una actividad docente más reflexiva, donde el alumno debe abordar un proceso de aprendizaje más complejo, sobre un tema concreto, y que culmina con la entrega de un informe o proyecto, siendo frecuente que además se exija su exposición pública ante el resto de estudiantes o ante una comisión académica integrada por profesores. Este trabajo de investigación o desarrollo se valora teniendo como referencia la aplicación de los conocimientos teóricos o prácticos que el alumno haya adquirido durante el proceso de evaluación docente, así como las habilidades relacionadas con la profesión que el alumno demuestre con la entrega del trabajo.

Precisamente, el sistema de enseñanza superior vigente en España hasta la implantación de las Experiencias Pilotos del Eurocrédito, circunscribía el proceso de evaluación a la elaboración de uno o varios exámenes, según se tratara de asignaturas cuatrimestrales o anuales; mientras que los trabajos de investigación o desarrollo como herramienta de evaluación final han sido utilizados en algunas Titulaciones (proyectos de fin de carrera en la rama técnica), pero sobre todo como prueba final en los estudios Post-Grado (Tesina y Tesis Doctoral).

De acuerdo con la afirmación anterior, las asignaturas de Derecho del Trabajo impartidas en la Diplomatura de Relaciones Laborales, y que integran en la actualidad el Grado de Relaciones Laborales y Recursos Humanos, han sido tradicionalmente evaluadas en nuestra Universidad mediante un examen final dividido en dos partes, una de tipo teórico, y otra de tipo práctico; en la realización de esta última parte los alumnos podían utilizar un compendio de legislación laboral para la resolución del supuesto planteado. Sin perjuicio de que las clases prácticas se emplearan para enseñar habilidades, a través de la aplicación de conocimientos teóricos y de la doctrina jurisprudencial, facilitando a los alumnos un conocimiento más cercano a la realidad cotidiana de las relaciones laborales, con la ventaja que ello comportaba, en cuanto a la resolución satisfactoria del examen, para quienes asistieran a estas sesiones. Así, estas sesiones prácticas, y la entrega en su caso de los supuestos, no computaban en la calificación final del alumno, salvo que la calificación obtenida en el examen se acercara a la nota del aprobado o la participación excepcional del alumno en alguna de las sesiones aconsejara su cómputo para incrementar la nota final obtenida. En otras palabras, el peso de la calificación obtenida por el alumno correspondía a la media 
entre los dos exámenes parciales o el resultado del examen final de la asignatura. Un sistema que se aplicaba de forma generalizada, siguiendo el modelo de enseñanza más común, con la única excepción de la asignatura de Prácticas integradas, donde los profesores habían ensayado algunos métodos de enseñanza más innovadores, basados en la simulación del caso, y donde el portafolios se empleaba en algunas actividades para soportar la enseñanza-aprendizaje (Mazuelos Flores, Borruel Toledo y Arena Viruez, 2010: pp. 272). En efecto, el enriquecimiento de profesores y alumnos en el proceso de aprendizaje, así como la necesidad de una mayor implicación de ambos, habían justificado que ocasionalmente se empleara el portafolios.

Frente al modelo de enseñanza tradicional antes descrito, el Espacio Europeo de Educación Superior ha impulsado importantes cambios en el sistema universitario español (De Miguel, 2005: 17), adoptando una importancia fundamental la evaluación de cada una de las fases del proceso formativo. De ahí que los profesores del Área de Derecho del Trabajo responsables de implantar este nuevo sistema durante los años de experiencias pilotos del ECTS, hubieran identificado la utilidad que este marco podía adquirir el portafolios como una de herramienta que permite evaluar el proceso de formación del estudiante (Rodríguez Santos y Gómez-Millán Herencia, 2006: 481).

De este cambio de modelo queda constancia en los documentos de trabajo para la implantación del Grado en Relaciones Labores y Recursos Humanos en nuestra Universidad, donde se refleja que la metodología debe ajustarse a una enseñanza más centrada en el alumno, lo que ha supuesto una distribución distinta de la docencia. En concreto, la Universidad Pablo de Olavide ha afrontado este cambio fijando una distribución regular de las clases magistrales a lo largo del curso académico, en sesiones de una hora y media; mientras que las prácticas y desarrollo ocupan un mínimo de siete sesiones con la distribución cronológica que los docentes elijan, en función de la idoneidad de impartir las sesiones en una fecha u otra. En este sentido, el manual o los manuales de la asignatura continúan desempeñando el papel de instrumento básico, sobre todo, para las clases magistrales; pero lo que interesa destacar en este momento es que las clases prácticas y de desarrollo implican una forma distinta de trabajo, normalmente en grupos, donde el alumno aprende competencias profesionales. A este respecto, el grado de reflexión y autonomía en el aprendizaje que se le exige al alumno, aspectos centrales en los nuevos sistemas de formación docente en la Universidad (Rodríguez Sánchez, 2011: 83), requiere un cambio tanto en la metodología como en la forma de evaluar. Es aquí donde encontramos la innovación más relevante que introduce el sistema europeo de enseñanza superior, dado que los sistemas de enseñanza tradicionales, basados normalmente en un examen único, al final del curso académico, facilitaban un tipo de aprendizaje basado en la mediatez de aprobar, pero sin profundizar en la importancia de adquirir conocimientos duraderos. En este sentido, las pruebas presenciales encajaban perfectamente con el 
sistema de enseñanza tradicional, pero no se tratan de las únicas formas de evaluar, ya que pueden igualmente existir otras formas complementarias a ésta, como son la elaboración de trabajos de investigación y desarrollo, más ajustadas a las ideas del Espacio Europeo de Educación Superior.

Fig. 1: Tipos de EVAluación docente

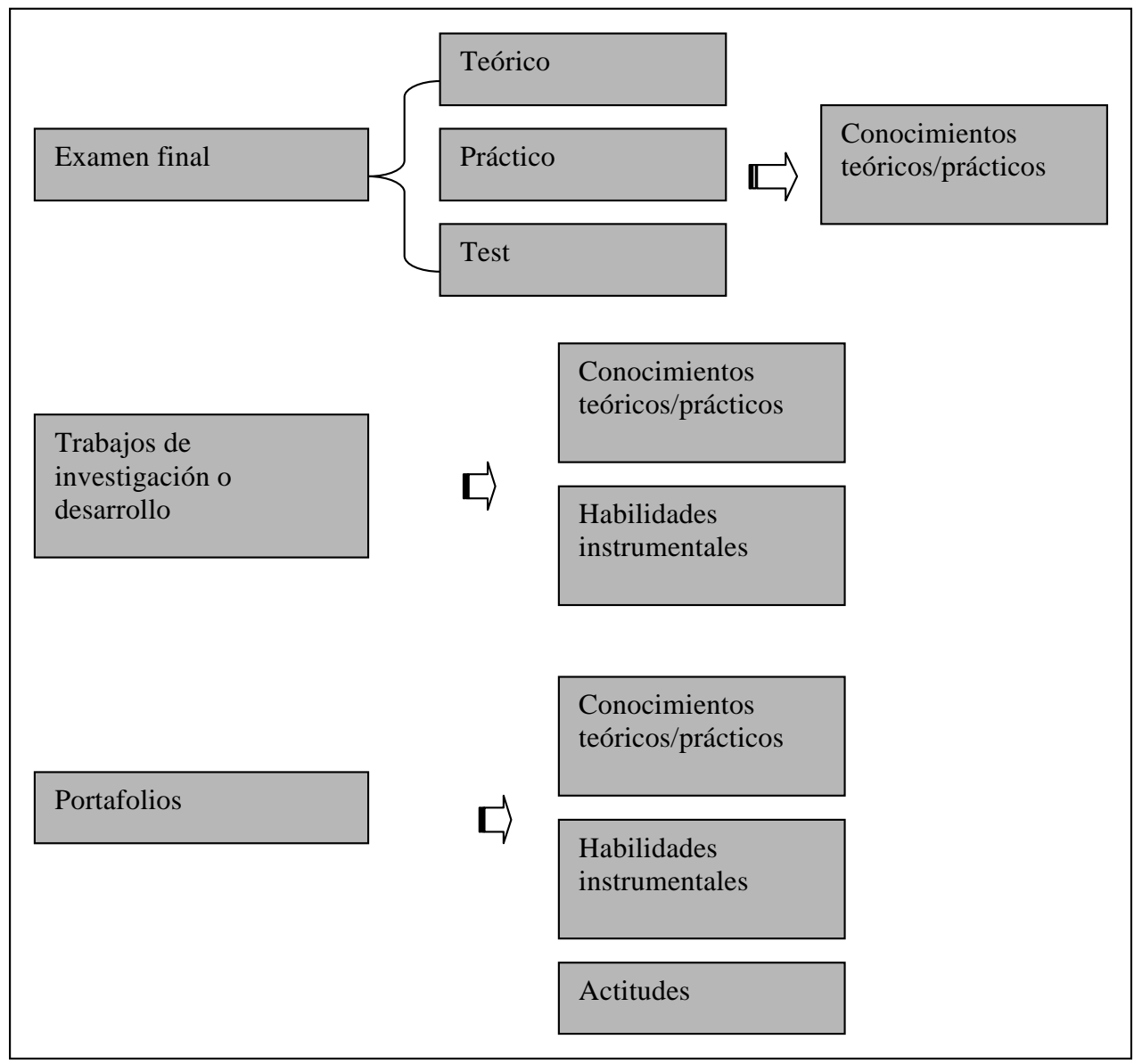

En efecto, el Espacio Europeo de Educación ha evidenciado la necesidad de emplear distintos instrumentos para evaluar los conocimientos de los alumnos, en tanto que el aprendizaje basado en las competencias (o en conocimientos profesionales y cualidades personales) se ha convertido en un de las ideas esenciales de este 
cambio de modelo (Zabalza Beraza, 2008: 79), con lo que ello supone en cuanto al peso del examen que deja de ser la única forma de evaluación, para comprender distintas pruebas que pueden surgir durante el proceso de aprendizaje.

Este aprendizaje más progresivo o procesual requiere igualmente de instrumentos que evidencien el transcurso del proceso formativo. Entre ellos, sin lugar a dudas, el portafolios ha adquirido una relevante presencia en la adaptación al sistema de educación superior. De ahí que, en la implantación del Grado en Relaciones Laborales, se haya rescatado la experiencia del portafolios, adquirida en las Prácticas Integradas o en los proyectos pilotos de ETCS.

\section{EL PORTAFOLIOS COMO HERRAMIENTA DE EVALUACIÓN EN EL GRADO DE RELACIONES LABORALES Y RECURSOS HUMANOS}

Ante la diversidad de tipos de portafolios que pueden existir, en el siguiente apartado se realiza una elaboración teórica del concepto de portafolio y sus características, con el fin de concretar la experiencia de portafolios en las asignaturas de Derecho del Trabajo pertenecientes al Grado de Relaciones Laborales y Recursos Humanos que se han impartido hasta la fecha utilizando como herramienta el portafolios (Introducción al Derecho del Trabajo), y de próxima implantación (La ordenación del empleo y de la igualdad en el trabajo).

\subsection{CONCEPTO Y CARACTERÍSTICAS}

Según el Diccionario de la Real Académica Española de la Lengua, el portafolios o portafolios es "una carpeta de mano para llevar libros, papeles" u otros documentos. Sin embargo, como herramienta de evaluación docente, el portafolios adquiere una dimensión mucho más amplia, con una funcionalidad que excede de la mera recopilación de materiales didácticos o de trabajos realizados por el profesor o los alumnos.

En este sentido, el portafolios se define como una colección de trabajos o evidencias en el que se incluyen los materiales utilizados, y donde se cuenta la historia de un proceso de enseñanza-aprendizaje, mediante la expresa mención de los esfuerzos, los logros y las dificultades encontradas, así como los instrumentos o medios de solución utilizados para superar estos obstáculos.

El portafolios, en este contexto, desempeña varias funciones. En primer lugar, tiene una finalidad "formativa", al permitir la transmisión de conocimientos teóricos y prácticos (enseñanza), así como la adquisición de competencias y habilidades para el alumno, a través de la enseñanza y el aprendizaje en una determinada materia (aprendizaje). En segundo lugar, es indudable su finalidad "evaluativa"; pues recoge las evidencias del proceso de aprendizaje que servirán para la evaluación de las competencias adquiridas fuera y dentro del aula. Esto hace que el portafolios se defina como 
una forma de enseñanza/aprendizaje, es decir, como un proceso de adquisición de conocimientos, y no tanto como una metodología específica o concreta de aprendizaje.

Todas estas funcionalidades definen al portafolios como un sistema de compilación de documentos con las siguientes características: 1) Es herramienta abierta y flexible que permite crear un marco de aprendizaje a medida, tratándose de un instrumento reflexión que facilita la fijación de conocimientos, concretando las etapas del proceso formativo. 2) El portafolios recoge competencias de diverso origen, entre las que se encuentran los conocimientos técnicos, las habilidades, las actitudes del alumno (Atienza Cerezo, 2009: 7), incorporando conocimientos tanto teóricos (Shulman, 2003: 55) como prácticos, ya sean contados por el profesor o por el alumno. 3) La información se recoge de una manera ordenada, lo que diferencian al portafolios de una carpeta o un Diario de campo (Barragán Sánchez, 2005: 6), en tanto que el proceso de aprendizaje se refleja cronológicamente siguiendo la lógica marcada por el profesor y el alumno, pero sin que el portafolios incorpore documentos carentes de justificación. 3) Las evidencias del proceso formativo que se guardan en el portafolios, siempre están sujetas a una constante revisión, en la que participan tanto los profesores como el resto de alumnos, siendo esencial la retroalimentación (Coromina, Romeo y Ruiz, 2011: 123). Por lo que su carácter dinámico o de revisión continua, como veremos en el próximo apartado, es una de las principales peculiaridades del portafolios, ya que lo distingue claramente de otras herramientas donde meramente se almacena información. 4) El portafolios, además, recoge los resultados del proceso formativo de una forma crítica, siendo una importante herramienta de reflexión (Alfageme González, 2007).

Fig. 2: CONCEPTO y CARACTERÍSTICAS DEL PORTAFOlios

\section{$\underline{\text { Concepto }}$}

Colección de trabajos, en continua revisión, donde se cuenta la historia de un proceso de enseñanza-aprendizaje, incluyendo las dificultades encontradas y los medios de solución utilizados

\section{$\underline{\text { Principales características }}$}

- Es una herramienta abierta

- Recoge conocimientos teóricos y prácticos

- Contiene materiales de manera ordenada

- Precisa la revisión de ideas

- Es un instrumento de reflexión 


\subsection{Tipos DE PORTAFOLIOS Y CONTENIDO MÍNIMO: HACIA EL PORTAFOLIOS MIXTO}

Entre los modelos o clases de portafolios se han distinguido aquellos denominados como sustantivos, por tratarse de una mera muestra o recopilación de evidencia, de otros denominados como formativos, a cuyo análisis se dedica la presente comunicación (Quero Gallego, 2008: 58). Asimismo, el portafolios puede también encaminarse al aprendizaje, a la enseñanza, a la evaluación, a la promoción y el desarrollo profesional. Esta última posibilidad ha dado origen a un importante debate sobre el uso de portafolios como forma de evaluar al alumno; en este sentido, se ha defendido la conveniencia de utilizar el portafolios como instrumento de enseñanza, y, adicionalmente, como instrumento de promoción y desarrollo profesional (Klenowski, 2004: 37).

1.- Centrándonos en el portafolios formativo, cada proceso de aprendizaje puede emplear uno o más tipos distintos de portafolios, creando como se decía un marco de aprendizaje a medida. En este sentido, pueden distinguirse tres modelos de portafolios: el portafolios del profesor, el portafolios del alumno y el portafolios mixto (Mazuelos Flores, Borruel Toledo y Arenas Viruez, 2010: 272).

En primer lugar, el portafolios de profesor se introdujo en las Universidades americanas como una forma de evaluar a los profesores, con el fin de que el docente se acerca a su asignatura de una manera crítica y reflexiva, a los efectos de constatar posibles carencias y, en su caso, resolver los obstáculos que encuentra en su dedicación como docente. Recogiendo esta idea, las Universidades españolas, en la adaptación al Espacio Europeo de Educación Superior, han incrementado el contenido de los programas docentes, introduciendo modificaciones que permiten, en nuestra opinión, avanzar hacia la implantación del portafolios del profesor.

En este punto, como exigencia del Vicerrectorado de Docencia y Convergencia Europea de la Universidad Pablo de Olavide, todas las asignaturas que se integran en el Grado de Relaciones Laborales y Recursos Humanos, en nuestra Universidad, cuentan con una Guía Docente, cuyos contenidos básicos son: identificación de la asignatura, porcentaje de las sesiones dedicadas a las enseñanzas básicas, las prácticas y desarrollo y las actividades dirigidas, responsable de la asignatura y equipo docente implicado, objetivos de la asignatura, temario, metodología de las sesiones, forma de evaluación, competencias a cuya formación se dirige y aportación de la asignatura al conjunto del programa formativo de la titulación, bibliografía general y recursos más adecuados a los objetivos propuestos. Esta Guía Docente además se completa con una parte específica, donde se concretan las actividades de contenido práctico en un cronograma que los alumnos pueden consultarse en una plataforma virtual (web-CT), así como la bibliografía específica para estas sesiones. Todos estos documentos tienen como meta orientar y tutorizar la labor de aprendizaje del alumnado, fijando una serie de pautas o criterios a partir de los cuales el estudiante irá adquiriendo las capacidades y habilidades que, posteriormente, serán 
evaluadas por el profesorado. Tratándose, en definitiva, de documentos que integran el contenido mínimo del portafolios.

Como herramienta indispensable, el portafolios del profesor incluye una ficha individual de cada alumno, donde se hacen las anotaciones de su proceso de aprendizaje, así como una ficha grupal por cada una de las actividades que se realizan de forma conjunta, en la que se anotan la participación de cada grupo y, en su caso, la aportación de cada uno de los alumnos en las actividades colectivas.

2.- En segundo lugar, el portafolios del alumno puede ser individual o grupal, según la clase de actividad o el diseño que el profesor elija para la impartición de la asignatura. El portafolios grupal se utiliza para aquellas actividades de carácter colectivo, y su finalidad es que los integrantes del equipo de trabajo incluyan, durante el transcurso del proceso de aprendizaje, sus propuestas de resolución de las actividades que sean comunes, así como otros documentos que se consideren de especial transcendencia en su proceso de aprendizaje. El portafolios grupal se elabora a partir de las tareas propuestas por el profesor, indicando al alumno la extensión del trabajo, pero con plena libertad en cuanto a la forma de presentación del trabajo. Junto a la propuesta de resolución, cada grupo de alumnos debe incluir un cuadro de tareas, en el que se especificará la distribución del trabajo entre los integrantes del grupo, la planificación del tiempo dedicado a la actividad, así como el grado de responsabilidad que finalmente han asumido cada uno de los integrantes. Por último, cada coordinador debe elaborar una memoria en la que ponga de manifiesto las dificultades encontradas, el tiempo de dedicación a la práctica, los comentarios referidos a cada integrante del grupo; este documento debe tener carácter confidencial.

Por otro lado, el portafolios tiene como finalidad soportar el proceso de aprendizaje de cada uno de los estudiantes, aunque se establezcan conexiones entre el portafolios individual y colectivo. Se integra, como mínimo, de los siguientes contenidos. En primer lugar, el Diario (o agenda de campo) es una de las herramientas básicas del portafolios, ya que el alumno recoge en él sus opiniones y valoraciones acerca de las técnicas utilizadas en el proceso de aprendizaje (desconcierto, motivación, agobio, entusiasmo), lo que permite tener una visión clara del proceso de aprendizaje del alumno, reflejando sus progresos, las dificultades encontradas, sus propuestas de mejora. En efecto, el Diario de campo permite al alumno identificar, al finalizar cada etapa formativa, sus carencias en las materias estudiadas, obligándole a buscar más información sobre aquellos aspectos menos tratados en su proceso formativo o que requieren de una formación adicional. De ahí que el Diario de campo tenga como finalidad hacer reflexionar al alumno sobre su proceso de aprendizaje, siendo un instrumento complementario (y no sustitutivo de la labor del profesor). Por otro lado, el Diario de campo facilita la labor docente, ya que el profesor puede conocer las dificultades a las que se han enfrentado los alumnos, permitiendo un nuevo diseño de la asignatura que supere los obstáculos iniciales. 
Asimismo, el Portafolios individual recogerá los trabajos que el alumno realice con la única ayuda del profesor, ya sean de actividades inicialmente propuestas por el docente encargado o responsable de la asignatura, o bien actividades realizadas a petición del alumno. En cada trabajo, se incluirá, con carácter indispensable, los resultados obtenidos a modo de conclusión y la bibliografía empleada.

Además, en el portafolios individual, los alumnos incluirán, tras finalizar cada práctica, un cuestionario sobre la actividad realizada. Estos cuestionarios se elaboran por el profesor, y persiguen, a través de preguntas teórico-prácticas, tener constancia documental de las competencias y habilidades que el estudiante ha adquirido con cada práctica.

En definitiva, el portafolios del alumno reflejará el trabajo desarrollado por el estudiante; en concreto, las descripción de las tareas realizadas, la información manejada, los resultados conseguidos, las dificultades encontradas durante el desarrollo del proceso, las cuestiones planteadas o resueltas en tutoría, las reuniones fuera y dentro del aula, así como la implicación del grupo en la elaboración de las tareas encomendadas, y el resultado final de cada trabajo.

3.- En último lugar, consideramos el Portafolios mixto como el instrumento ideal o la meta al que deberían aspirar tanto los docentes como los alumnos implicados en un proceso de enseñanza-aprendizaje concreto, sobre todo en la implantación de los Grados, donde los autores de este trabajo (que asumen docencia en el Grado de Relaciones Laborales y Recursos Humanos) ya han puesto en marcha algunas ideas en las que se basa esta herramienta.

En efecto, el Portafolios mixto, que sería el resultado o producto final de las entradas que realizan tanto profesores como alumnos durante el curso académico, estaría integrado por el portafolios del profesor, el portafolios individual y colectivo de los alumnos, así como por una memoria elaborada por el profesor-responsable de la asignatura, en la que se detallaran los objetivos iniciales, los instrumentos utilizados, las metas alcanzadas, las propuestas de modificación de los contenidos de la asignatura o de las metodologías aplicadas. Esta memoria, en definitiva, cerraría el Portafolios mixto.

Es preciso, en este sentido, destacar que el alumno también puede (y sería lo deseable) intervenir en el portafolios del profesor, proponiendo trabajos o actividades, e incluso aportando recursos que le hayan facilitado su proceso formativo. Al mismo tiempo, estas entradas también pueden realizarse entre los alumnos, lo que resulta de interés para generar un proceso de sinergias formativas entre los compañeros de un mismo grupo, favoreciendo competencias específicas, como son el trabajo colaborativo. Este sistema se ha utilizado para la enseñanza de algunos temas donde uno/varios estudiantes, de manera voluntaria, asumen la responsabilidad de profundizar en una materia, como especialistas, lo que les permite desempeñar tutorizar a sus compañeros, siempre contando con la supervisión del profesor. 
Sin embargo, el portafolios mixto no se ha empleado en toda su extensión, en buena medida porque su implantación precisa de herramientas y recursos que soporten la puesta en marcha de este sistema novedoso, cuya aplicación privaría de sentido a otros sistemas de evaluación, como el examen, que siguen contando con un peso significativo en la evaluación del alumno.

Fig. 3: Tipos DE PORTAFOlios Y CONTENIDO BÁSICO
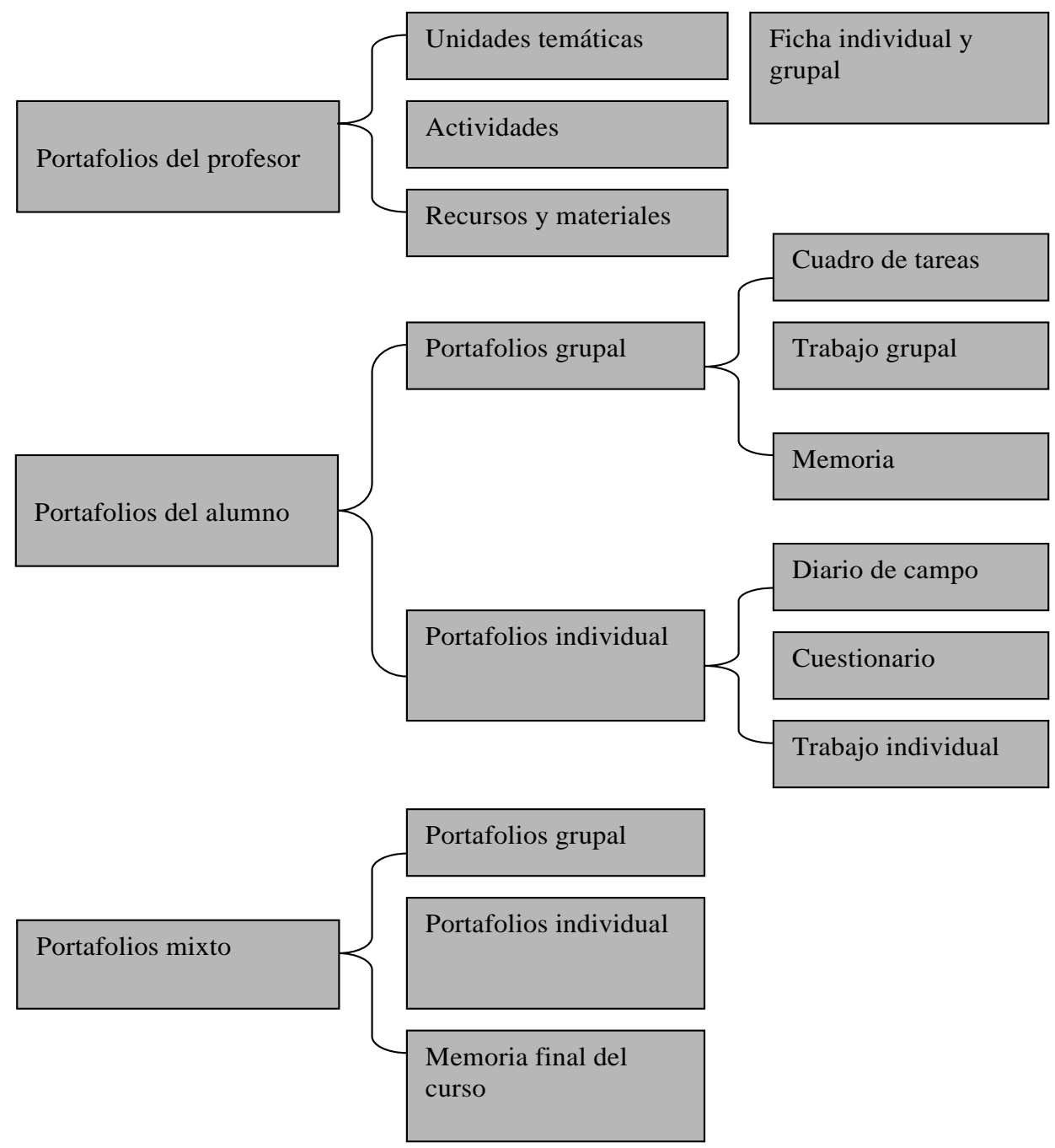


\subsection{Proceso de Elaboración}

El portafolios puede presentarse en distintos soportes. Nada obstaculiza el mantenimiento del clásico soporte papel, si bien es cierto que las experiencias de implantación apuntan hacia la utilización de documentos electrónicos. En este sentido, el portafolios electrónico facilita el seguimiento del profesor, dado que abre la posibilidad de consultar el proceso de aprendizaje a medida que éste toma forma. Como modalidad específica, en algunas Universidades se plantea el desarrollo del portafolio digital, empleando como soporte un sistema informático común, que permitirá el acceso a todos los docentes y alumnos, y en el que se podrán almacenar todos los datos del proceso de aprendizaje. A pesar de lo dicho, la digitalización de documentos ha sido utilizado fundamentalmente para el portafolios del profesor; los alumnos continúan haciendo un uso mayoritario del soporte papel, entregando al final de curso la carpeta con todos los materiales para que el profesor evalúe el proceso de aprendizaje.

El portafolios consta de dos partes esenciales, la parte que refleja el proceso de aprendizaje, de un lado, y de otro lado, la parte en la que se contiene el resultado de la experiencia formativa (Coromina, Romeo y Ruiz, 2011: 123). Por lo que se refiere a la parte que refleja el proceso de aprendizaje, el profesor debe poner a disposición del alumno el contenido íntegro de su portafolios al inicio del curso académico, facilitando con ello la transparencia del proceso de aprendizaje. Los alumnos implicados en la elaboración del portafolios, por su parte, deben realizar una preparación de la asignatura antes de iniciar el curso académico, que pasa por un conocimiento suficiente del contenido del portafolios del profesor, en concreto, de la parte referida al objetivo de la asignatura, la metodología y la forma de evaluación, el cronograma y los finalidades que se persiguen con cada una de las actividades.

Una vez iniciado el curso académico, el alumno debe crear carpetas (ya sea en soporte electrónico o físico) que le permitan la elaboración de las dos partes que, según se ha dicho, constituyen el contenido mínimo del portafolio individual y grupal. Dependiendo de la actividad, se utilizará cada uno de los portafolios, haciendo las anotaciones pertinentes. En cualquier caso, el proceso que debe seguir el alumno antes de realizar cualquier entrada debe superar las siguientes fases: 1) lectura de las actividades propuestas y de los objetivos, tanto generales de la asignatura como particulares de la tarea que emprende; 2) recogida de las evidencias o datos que se estimen precisos para realizar la actividad; 3) puesta en común de las evidencias, con el profesor, y en el caso de tratarse del portafolios grupal, con el resto de compañeros; 4) elaboración del trabajo; 5) cierre del trabajo, incluyendo las conclusiones. Este proceso debe quedar recogido en el portafolios, realizándose por cada actividad conforme al cronograma incluido en la planificación del curso académico. 
Por su parte, el profesor revisa el portafolios y evalúa al estudiante valorando especialmente los documentos aportados en los que se aprecie el interés del alumno/a por auto-formarse y que revelen sus inquietudes personales dentro de su propio proceso de aprendizaje profesional. El alumno, por su parte, puede participar en dicho proceso evaluativo, discutiendo con el profesor el significado de sus trabajos. Así, el procedimiento para la revisión y evaluación del portafolios será el siguiente: la carpeta se le entregará al profesor/a tras finalizar cada práctica; una vez revisada por el profesor/a y evaluado su contenido, se le devolverá al estudiante para que incluya los documentos referidos a la siguiente práctica. Este procedimiento deberá reiterarse cada vez que se realice una nueva práctica, realizando el alumno las correspondientes anotaciones tanto del trabajo inicialmente entregado como de las revisiones que realice el docente, con la oportuna valoración sobre las dificultades que ha encontrado, las herramientas de solución, los conocimientos aprendidos y las deficiencias que encuentre en su proceso.

En concreto, por lo que se refiere a las actividades prácticas y de desarrollo, en la experiencia piloto de ECTS denominados como Talleres o Seminarios, y cuyo objetivo es profundizar sobre los conocimientos prácticos, los profesores proponían una actividad que los alumnos resolvían antes de acudir a la sesión de clase, quedando reflejado este proceso en el portafolios. Una vez en el aula se discutía la actividad, y los alumnos debían asimismo revisar el trabajo realizado para incluirlo en el portafolios. Como ejemplo, se relaciona el contenido de estas actividades desarrolladas durante el pasado curso académico: 1) uso de los recursos electrónicos de la Biblioteca de la Universidad, 2) búsqueda de resoluciones judiciales (bases de datos jurídicas), 3) análisis de estructura de una sentencia: los antecedentes de hecho, los fundamentos jurídicos y el fallo o pronunciamiento judicial, 4) cita y aplicabilidad de resoluciones judiciales, 5) estudio de un caso práctico: detección de los elemento de discusión y propuesta de resolución, 6) búsqueda de bibliografía, consulta de revistas especializadas: la doctrina científica.

Otra de las actividades, que también debían detallarse en el portafolios, eran las actividades de debate y puesta en común de materias relacionadas con las clases, que los alumnos debían igualmente preparar antes de la sesión, contando para ello con el apoyo del profesor. Con carácter orientativo, estas sesiones trataban sobre: "la acción positiva", "el precontrato de trabajo y "sus efectos jurídicos", "la intimidad del trabajador versus el poder de dirección del empresario", "derechos fundamentales de lo trabajadores". El alumno tenía que incluir una memoria antes del debate, incluyendo posteriormente las observaciones que creyera oportunas.

Asimismo, el alumno debía incluir en el portafolios otras actividades, como las visitas o las sesiones de coloquios, que igualmente contribuyeran a enriquecer su proceso formativo, haciendo las pertinentes observaciones y reflexiones, en el Diario de campo, sobre los conocimientos adquiridos en su transcurso. 


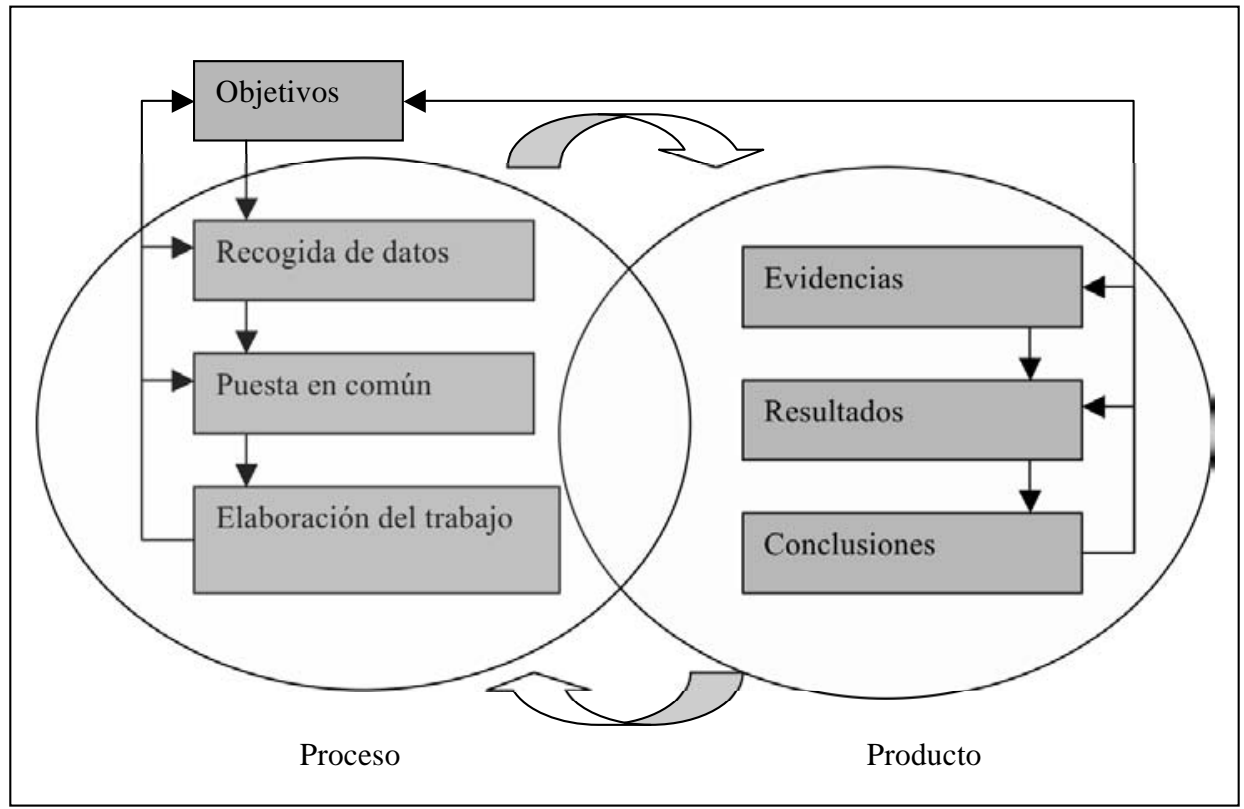

El alumno debe completar esta carpeta durante todo el curso, pudiendo seleccionar libremente los documentos que incluirá en el portafolios, siendo objeto de valoración por el profesorado de forma conjunta. No existe, en este sentido, un criterio sobre qué tipo de evidencias o muestras deben incluirse en el portafolios, pero se sabe que el alumno debe decidir entre aquellas que permitan una valoración más ajustada de su proceso formativo. Esto implica que el profesor puede diseñar un proceso de aprendizaje abierto donde pueden existir actividades alternativas, con una valoración distinta según el grado de dificultad, sin que la calificación final pierda objetividad.

Como existen distintos tipos de portafolios, una de las cuestiones que han de afrontar los docentes es la conexión entre ellos, siendo la tutoría o la revisión elementos esenciales que enriquecen el proceso de aprendizaje; y, por tanto, del portafolios.

En definitiva, en el portafolios, se estructuran con un orden las tareas que van a ser objeto de evaluación, aplicando una información previa, construyendo respuestas y explicando el proceso que les ha llevado a la elección de una determinada respuesta. 


\section{CONCLUSIONES}

Entre los argumentos a favor de la utilización del portafolios como herramienta de evaluación estaría los efectos potenciadores que dicha posibilidad proyecta sobre el auto-aprendizaje del estudiante. Incrementar la capacidad del estudiante para protagonizar el proceso de adquisición de conocimientos resulta un elemento clave en la mejora de la enseñanza universitaria que debe extenderse al conjunto de la vida profesional de éste. Adicionalmente, el fomento del espíritu o de la aptitud crítica del estudiante frente a los instrumentos, prácticas, reglas e instituciones jurídico-laborales constituye un elemento esencial en un entorno de enseñanza como el definido en el espacio europeo de educación superior. La reiteración de informaciones, afirmaciones, reglas y contenidos, preelaborados por la doctrina científica hasta la fecha, limitan la creatividad del estudiante y reduce las posibilidades formativas de quienes deben contribuir de manera decidida a superar los modelos ahora vigentes. En este sentido, el portafolios promociona desde un momento temprano el desarrollo de estas capacidades, situando al estudiante en el centro del proceso de aprendizaje, mediante la recuperación de un determinado grado de protagonismo en la construcción de su proceso de aprendizaje y cooperando con sus compañeros en el progreso de un colectivo que le permite un mayor nivel de integración e identificación con sus compañeros.

Desde nuestro punto de vista, el sistema tradicional de evaluación hace girar el conjunto del proceso de enseñanza aprendizaje sobre la superación de una prueba escrita. Esta realidad provoca efectos perversos sobre la enseñanza universitaria en su conjunto y sobre el nivel de formación de los titulados. En el ideario colectivo se mantiene la idea de que realizar unos estudios universitarios consiste fundamentalmente en superar un número determinado de exámenes, para lo cual el estudiante debe memorizar determinada información contenida en manuales o, en el peor de los casos, en apuntes de clase, en no pocas ocasiones trasmitidos de curso en curso. De esta forma, se desnaturaliza la enseñanza universitaria, hasta el punto de que resulta igualmente común la idea de que donde verdaderamente se aprenden las competencias y aptitudes para desarrollar cualquier profesión en la empresa. Si esto es realmente así, en nada se justifican los largos años que los estudiantes deben pasar en la Universidad española y los importantes recursos públicos empleados con dicho objetivo. Si fuésemos capaces de limitar y, a medio plazo, eliminar los exámenes tradicionales, colocaríamos al estudiante ante una realidad bien diferente. Estudiar una carrera universitaria exigiría un esfuerzo seguramente mayor, pero los resultados obtenidos casi con toda certeza podrían ser también mejores. Si somos capaces de trasmitir al estudiante que su futuro profesional comienza con su presente universitario, que desde el primer curso debe iniciar el proceso de aprendizaje de las competencias y capacidades que le permitirán desarrollar una carrera profesional en la que podrá desempeñar distintos puestos de trabajo o profesiones y, sobre todo, que la responsabilidad de elaborar y recorrer la 
hoja de ruta adecuada es compartida con sus profesores y compañeros, la Universidad española daría un importante paso adelante.

En este sentido, la utilización del portafolios como instrumento en la docencia y en la evaluación facilita la consecución de estos fines desde el primer momento, colocando desde el inicio del curso al estudiante ante una serie de responsabilidades que difícilmente podrá rehuir. El portafolios permite una tutela más intensa e inmediata del proceso de aprendizaje por parte del profesor, lo que facilita la evaluación del desarrollo del proceso, pero también del resultado final que se alcanza. Las deficiencias del proceso de aprendizaje quedan puestas de manifiesto en los momentos iniciales del curso, de manera que si existe voluntad por las partes, pueden ser solventadas de manera eficaz. La no superación de la asignatura no se produce a la mitad del curso o al finalizar éste, la no superación de la asignatura es el resultado de un proceso que ahora cobra una transparencia superior y, con ello, pueden ponerse en marcha medidas preventivas antes de que la situación se deteriore.

Por otra parte, el portafolios facilita el desarrollo de tareas de forma colaborativa, con la implicación de grupos de estudiantes en el proceso, mediante distintas técnicas que permiten detectar el grado de implicación de los alumnos. Conviene recordar que el graduado, en la mayoría de su actividad como profesional del Grado en Relaciones Laborales y Recursos Humanos, tendrá que colaborar con otras personas: compañeros de trabajo, jefes, subordinados, clientes, etc.; un universo de cooperación que no puede ser obviado durante el proceso de aprendizaje. Dicho en otras palabras, el graduado no es un opositor y, si lo ha sido, superado este proceso debe integrarse en una organización pública o privada en la que desempeñará su actividad. Por ello, no tiene mucho sentido centrar el proceso de enseñanza aprendizaje en la individualidad del estudiante, en la competencia con sus compañeros, en la maximización del esfuerzo individual. Por el contrario, si la realidad del mundo profesional es la colaboración, el estudiante debe formarse también en una serie de competencias colaborativas, vinculadas a las asignaturas que debe cursar. Por ello, el trabajo en grupo, la colaboración con sus compañeros y con el profesorado resulta una herramienta clave en la formación. Aunque puedan existir diferentes herramientas de aprendizaje y evaluación que contemplan el trabajo en grupo, el portafolios permite registrar con prontitud los errores u obstáculos comunes, facilitando la inmediata intervención del profesorado. Con la elaboración de actividades prácticas en común, los estudiantes pueden ejercitar sus facultades de liderazgo, negociación, colaboración, resolución de conflictos, integración, cohesión, solidaridad y permitirá a estos disfrutar de las ventajas que reporta la consecución de objetivos comunes.

Por otra parte, el portafolios individual permite ajustar el sistema de enseñanza a las diferentes necesidades de cada estudiante, personalizando los itinerarios formativos de cada alumno. El portafolios individual ofrece una información detallada sobre el aprendizaje; implica un aprendizaje colaborativo (profesor/alumno) enriqueciendo el proceso de 
aprendizaje; permite reflejar los avances en el proceso de aprendizaje; incita a un mayor nivel de compromiso del estudiante con el proceso de formación, proporciona información suficiente, amplia y profunda, que permite valorar las competencias de conocimiento y las actitudes de los alumnos, fomenta el espíritu crítico y la capacidad de reflexión. Todo ello permite al profesorado facilitar que cada estudiante pueda alcanzar el nivel que desee o para el que en su momento vital se encuentre capacitado. Cada alumno puede explorar, dentro de los parámetros marcados por el programa de la asignatura, los aspectos que considere más interesantes, aquellos que provoque en su interior un mayor interés, una mayor apetencia intelectual o considere más útil para sus perspectivas profesionales.

De lo dicho hasta aquí pudiera inferirse la necesidad de realizar una apuesta radical por el modelo de enseñanza y evaluación presentado. Pero la implantación de este método presenta importantes dificultades que deben ser igualmente puestas de manifiesto. De un lado, a nadie se le escapa que la utilización del portafolios, en la forma que ha sido presentada hasta aquí, genera un considerable aumento de las labores del docente. El tiempo actualmente dedicado a la docencia se vería por tanto incrementado de manera notable, tanto en la preparación de actividades como en su revisión o discusión con los estudiantes. En el actual caos presupuestario, frente a un previsible aumento del número de alumnos por grupos docentes, poner en práctica este método con todas sus consecuencias es una posibilidad escasamente realista. Particularmente negativo nos parecería la tentación de poner en práctica estos métodos de evaluación sin los recursos adecuados; en tal caso, puede que el portafolio se reduzca a un sistema de aprendizaje y evaluación que fomente valores contrarios a los aquí planteados y, en la práctica, tanto el profesor como el alumno tan sólo recopilen una serie de materiales, perdiendo toda función como herramienta de evaluación docente.

Quizás por ello, a pesar de que se ha venido implementado el espacio europeo de educación en todas las titulaciones, en nuestra universidad siga siendo frecuente que el profesorado acuda a los mecanismos de evaluación tradicionales. Desde nuestro punto de vista, el cambio que requiere el modelo de enseñanza universitaria debe ser asumido de forma generalizada por el claustro de las universidades y, en todo caso, por el profesorado de la titulación, pues en caso contrario, los estudiantes reciben un mensaje contradictorio, se incorporan a una universidad excesivamente heterogénea, que funciona al menos a dos velocidades, circunstancia que les obliga a aventurarse en el descubrimiento de un nuevo proceso de enseñanza aprendizaje, mientras permanecen con un pie en el modelo tradicional. De esta forma, parece más que obvio, es difícil caminar.

\section{BIBLIOGRAFÍA}

ATIENZA CEREZO, E.: "El portafolios del profesor como instrumento de auto-formación", Revista de didáctica, no 9, 2009, pp. 1-19. 
ALFAGEME GONZÁLEZ, M.B.: "El portafolios reflexivo: metodología didáctica en el EES", Educatio XXI: Revista de la Facultad de Educación, no 25, 2007, pp. 209-228.

BARRAGÁN SÁNCHEZ, R.: "El Portafolios, metodología de evaluación y aprendizaje de cara al nuevo Espacio Europeo de Educación Superior. Una experiencia práctica en la Universidad de Sevilla", Revista Latinoamericana de tecnología educativa, volumen 4, número 1, 2005, pp. 121-140.

BORDAS, M.I., y CABRERA, F.A.:"Estrategias de evaluación de los aprendizajes centrados en el proceso", Revista de Pedagogía, n 218, pp. 25-48.

COROMINA, J.; ROMEU, J.; RUIZ, F.: "Portafolio digital de aprendizaje", Intangible Capital, $\mathrm{n}^{\mathrm{o}}$ 1, 2011, pp. 116-142.

DE MIGUEL, M.: "Cambio de paradigma metodológico en la Educación Superior. Exigencias que conlleva”, Cuadernos de integración Europea, no 2, 2005, pp. 16-27.

KLENOWSKI, V.: Desarrollo de portafolios para el aprendizaje y la evaluación, Narcea, Madrid, 2004, pp. 21-38.

MAZUELOS FLORES, M.D.; BORRUEL TOLEDO, P. y ARENAS VIRUEZ, M.: "Los sistemas de innovación docente: aplicación práctica del portafolio como herramienta de cambio del sistema de trabajo", en VV.AA.: COTINO HUESO, L. y PRESNO LINERA, M.: Innovación educativa en el Derecho Constitucional. Recursos, reflexiones y experiencias de los docentes, Valencia, 2010, pp. 272.

RODRÍGUEZ SÁNCHEZ, M.: "Metodologías docentes en el EES: de la clase magistral al portafolio", Tendencias pedagógicas, no 17, 2011, pp. 83-103.

RODRÍGUEZ SANTOS, E. y GÓMEZ-MILLÁN HERENCIA, M.J.: "Programación de la asignatura Derecho del Trabajo en el marco ECTS", VV.AA.: Jornadas de trabajo sobre experiencias pilotos de implantación del crédito europeo en las Universidades Andaluzas, Cádiz, 2006, pp. 477-482.

SHULMAN, L.: "Portafolios del docente: una actividad teórica", en VV.AA.: El uso de portafolios: propuestas para un nuevo profesionalismo docente, Amorrortu, Buenos Aires-Madrid, 2003: pp. 55 y ss.

VILLARINI, A., "Primer Seminario taller sobre fundamentos y principios de evaluación auténtica. Facultad Autónoma de Santo Domingo, 1996.

QUERO GALLEGO, M.: "La práctica del portafolios como herramienta para el reconocimiento de competencias adquiridas en el comercio justo", Educar, 2008, pp. 53-61.

ZABALZA BERAZA, M.A.: "El trabajo por competencias en la enseñaza universitaria", en VV.AA.: El nuevo perfil del profesor universitarios en el EEES: claves para la renovación metodológica, Universidad Europea Miguel Hernández, Valladolid, 2008, pp. 79-113. 
ANEXO I: MODELO DE DIARIO DE CAMPO I ${ }^{1}$

Fecha:

Práctica y Desarrollo $\mathrm{n}^{\circ}$ :

¿Qué he aprendido hoy?. ¿De las explicaciones recibidas qué es lo que tengo ahora más claro?.

¿Qué ideas de las aportadas por el profesor/a o por mis compañeros/as de equipo me parecen más interesantes?.

¿Qué es lo que no he entendido bien?

¿Qué tendría que hacer para clarificar los aspectos sobre los que carezco de conocimiento previo?

1. Para la implantación de este instrumento, se utilizarán, entre otras, las ideas (en concreto, las preguntas formuladas a título ilustrativo) aportadas en el artículo elaborado por BORDAS, M.I., y CABRERA, F.A., en su artículo "Estrategias de evaluación de los aprendizajes centrados en el proceso", Revista de Pedagogía, no 218, pp. 25-48. 
¿Qué herramientas o instrumentos tengo a mi alcance para cubrir mis lagunas formativas?.

¿Qué dificultades encuentro para la profundizar sobre las ideas que me han sido aportadas?.

¿Cuál ha sido mi participación hoy en clase?.

Mi valoración de las competencias y habilidades profesionales desarrolladas en la sesión lectiva (en una escala de 1 a 5) ha sido de: 
MODELO DE DIARIO DE CAMPO II ${ }^{2}$

Periodicidad: Al finalizar cada Práctica y Desarrollo.

Fecha:

Práctica y Desarrollo n :

¿Me ha resultado interesante la realización de esta Práctica?. En caso negativo, ¿por qué?.

¿Qué me ha aportado su realización, en términos de competencias y habilidades profesionales?.

¿Cuánto tiempo le he dedicado a la realización de la Práctica?.

2. Para la redacción de este modelo, se ha recurrido, de una forma muy general, al esquema propuesto por VILLARINI, A., "Primer Seminario taller sobre fundamentos y principios de evaluación auténtica. Facultad Autónoma de Santo Domingo, 1996; para este autor, la autorreflexión del alumno/a debe estructurarse en tres apartados: cuestiones referidas a la autorregulación, al control de la acción y al control del conocimiento. 
¿He planificado mi tiempo en función de las tareas encomendadas?.

¿He sabido identificar la problemática que la práctica planteada?.

¿Qué procedimientos he utilizado para la resolución de las cuestiones planteadas?.

Mi aportación al grupo de trabajo, (en una escala de 1 a 5), en relación a los siguientes aspectos, ha sido de:

Responsabilidad:

Conocimientos técnicos:

Ética:

Competencias: 


\section{ANEXO II: MODELO DE CUESTIONARIO}

Práctica y Desarrollo $n^{\circ}$ :

Apellidos y Nombre:

D.N.I o pasaporte:

Identificación del Grupo:

1.- ¿A qué tipo de trabajadores se refiere el artículo 1 de la LOLS?

2.- ¿Quiénes están excluidos del ejercicio de la libertad sindical?

3.- ¿Pueden los parados sindicarse?

4.- ¿Cómo se constituye un sindicato?

5.- ¿Cómo se mide la representatividad del sindicato?

6.- ¿Puede el empresario recaudar las cuotas sindicales para transferirlas al sindicato? Razone la respuesta. 
ANEXO III: MODELO DE FICHA GRUPAL

Fig. 5 Ficha GRUPAL ANVERSO

№ del Grupo:
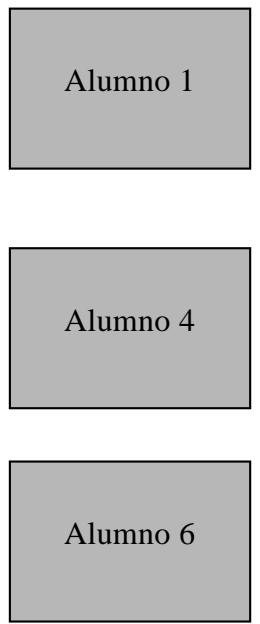

№ Prácticas y Desarrollo:
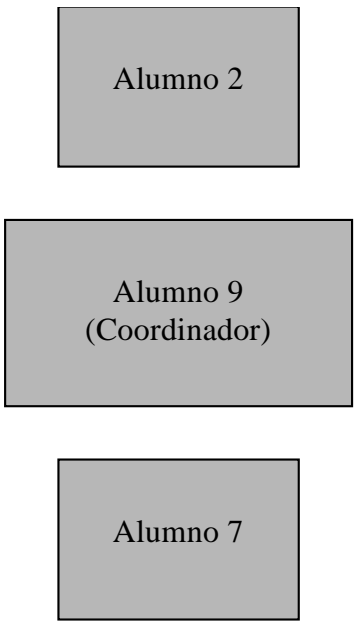

Fecha:

Alumno 3

Alumno 5

Alumno 8

Fig. 6 Ficha GRUPAL REVERSO

\begin{tabular}{|l|l|l|l|l|l|}
\hline & $\begin{array}{c}\text { A pellidos y } \\
\text { nombre }\end{array}$ & D.N.I. & $\begin{array}{c}\text { Firma de } \\
\text { entrada }\end{array}$ & $\begin{array}{c}\text { Firma de } \\
\text { salida }\end{array}$ & Observaciones \\
\hline $\mathbf{1}$ & & & & & \\
\hline $\mathbf{2}$ & & & & & \\
\hline $\mathbf{3}$ & & & & & \\
\hline $\mathbf{4}$ & & & & & \\
\hline $\mathbf{5}$ & & & & & \\
\hline $\mathbf{6}$ & & & & & \\
\hline $\mathbf{7}$ & & & & & \\
\hline $\mathbf{8}$ & & & & & \\
\hline $\mathbf{9}$ & & & & & \\
\hline
\end{tabular}

